\title{
Atomistic Explanation of Shear-Induced Amorphous Band Formation in Boron Carbide
}

\author{
Qi An, William A. Goddard III, and Tao Cheng \\ Materials and Process Simulation Center, California Institute of Technology, Pasadena, California 91125, USA \\ (Received 12 May 2014; published 28 August 2014)
}

\begin{abstract}
Boron carbide $\left(\mathrm{B}_{4} \mathrm{C}\right)$ is very hard, but its applications are hindered by stress-induced amorphous band formation. To explain this behavior, we used density function theory (Perdew-Burke-Ernzerhof flavor) to examine the response to shear along 11 plausible slip systems. We found that the $(01 \overline{1} \overline{1}) /\langle\overline{1} 101\rangle$ slip system has the lowest shear strength (consistent with previous experimental studies) and that this slip leads to a unique plastic deformation before failure in which a boron-carbon bond between neighboring icosahedral clusters breaks to form a carbon lone pair (Lewis base) on the $\mathrm{C}$ within the icosahedron. Further shear then leads this Lewis base $\mathrm{C}$ to form a new bond with the Lewis acidic $\mathrm{B}$ in the middle of a CBC chain. This then initiates destruction of this icosahedron. The result is the amorphous structure observed experimentally. We suggest how this insight could be used to strengthen $\mathrm{B}_{4} \mathrm{C}$.
\end{abstract}

DOI: 10.1103/PhysRevLett.113.095501

PACS numbers: 61.50.Ks, 62.20.M-, 64.70.-p, 82.40.Fp

Boron carbide $\left(\mathrm{B}_{4} \mathrm{C}\right)$ exhibits such novel properties as high melting temperature, high thermal stability, high hardness, low density, and high abrasion resistance [1-11]. The combination of these properties makes it widely useful in refractory applications, as abrasive powders, in body armor, and as a neutron radiation absorbent [1-4]. However, the brittle failure under impact exhibited by $\mathrm{B}_{4} \mathrm{C}$ prevents the wide insertion of boron carbide into extended engineering applications. Although $\mathrm{B}_{4} \mathrm{C}$ has a high Hugoniot elastic limit (HEL) of 17-20 GPa, approximately twice that of normal ceramics, it fractures easily just above the HEL at high impact velocities and pressures [12-15]. Chen et al. reported the observation of shock-induced local amorphization bands that might be responsible for the low fracture toughness of $\mathrm{B}_{4} \mathrm{C}$ [6]. In addition, amorphization bands had been observed in nanoindentation and scratch experiments, where the loading rate is much lower than for dynamical shock loading [16-19]. Particularly, recent nanoindentation experiments revealed amorphous shear bands along the $(01 \overline{1} \overline{1})$ plane [19]. Indeed, the same amorphous shear band has been observed experimentally in boron suboxide $\left(\mathrm{B}_{6} \mathrm{O}\right)$ [20]. An attempt to understand this through Gibbs free-energy calculations based on density functional theory suggested that the $\left(\mathrm{B}_{12}\right) \mathrm{CCC}$ structure provides a possible source of failure of boron carbide just above the HEL [7]. However, despite these extensive experimental and theoretical efforts, the atomistic mechanism underlying pressure-induced amorphization and phase transitions of boron carbide is still not known [3].

Stressed materials normally dissipate the accumulating elastic energy through plastic deformation that is mediated by dislocation slip and deformation twinning in metallic systems. Unlike these general deformation mechanisms in conventional metallic materials, we find that boron carbide deforms by atomic scale amorphous band formation, a particular mode of deformation that causes the material to be quite brittle, failing catastrophically due to uninhibited propagation of the amorphous bands. This amorphous band formation deformation mechanism makes the $\mathrm{B}_{4} \mathrm{C}$ a very good example to show that ultrahigh elastic stiffness does not necessarily translate into ultrahardness. Thus, this mechanism of amorphous band formation in $\mathrm{B}_{4} \mathrm{C}$ is essential to understand the deformation mechanism on covalent solids. In addition, understanding the role of bonding changes during deformation is essential to improve the material properties for hard covalent solids as indicated by several recent papers [21-24]. To understand these issues, it is necessary to examine the structural and bonding character in boron carbide under stresses. Previous simulations studied only hydrostatic or uniaxial compression on arbitrary directions $[8,25]$. We find that the directional nature of amorphous band formation requires study of a variety of deformation paths.

In order to obtain this atomistic understanding of the failure of $\mathrm{B}_{4} \mathrm{C}$, we applied finite shear deformation until failure along 11 different slip systems of $\mathrm{B}_{4} \mathrm{C}$ using density functional theory (DFT) at the Perdew-Burke-Ernzerhof (PBE) functional level. We found that the $(01 \overline{1} \overline{1}) /\langle\overline{1} 101\rangle$ slip system has the lowest shear strength among these 11 cases, leading to failure after a shear stress of $37.2 \mathrm{GPa}$ at 0.331 strain. This explains the recent experimental observation [19] that the shear-induced amorphization preferentially takes place along the crystal plane $(01 \overline{1} \overline{1})$ under nanoindentation.

We then extracted the atomistic mechanism of the failure along this particular slip system. We found that the failure process of $\mathrm{B}_{4} \mathrm{C}$ is directly related to the interaction of the $\mathrm{CBC}$ chain with the icosahedron. The key steps of the failure are (1) first, a B-C bond is broken between neighboring icosahedra; (2) this results in a lone pair on the $\mathrm{C}$ (a Lewis base) and an empty orbital on the $\mathrm{B}$; (3) this lone pair on the $\mathrm{C}$ then interacts with the middle boron in the $\mathrm{CBC}$ chain, which formally has $\mathrm{C}^{-} \mathrm{B}^{+}-\mathrm{C}$ character making this chain B a Lewis acid; (4) the formation of the 
Lewis base-Lewis acid bond from the icosahedral $\mathrm{C}$ to the chain $\mathrm{B}$ reduces the bond orders of the five intraicosahedral B bonds to this C, stretching them.

The result is decomposition of the icosahedral structure, leading to amorphization.

In order to clarify these issues further, we next give some background information on these $\mathrm{B}_{4} \mathrm{C}$ systems. The boron carbide structure $\mathrm{B}_{4} \mathrm{C}$ consists of 12-atom icosahedral clusters at the vertices of a rhombohedral lattice that are cross-linked by atomic chains of three atoms along the trigonal axis (the $c$ axis of the hexagonal representation) of the rhombohedral unit cell shown in Fig. S1(a) of the Supplemental Material (SM [26]) [3,27]. Each icosahedral cluster has two types of chemically distinct sites: six equatorial sites that each connect to a chain and six polar sites that each connect directly to another icosahedron. However, the distribution of the carbon atoms between the icosahedral cluster and the three-atom chain is not known experimentally. This leads to two plausible chain structures: $\mathrm{C}-\mathrm{B}-\mathrm{C}$ in which each $\mathrm{C}$ bonds to three icosahedra making the $\mathrm{B}$ formally $\mathrm{B}^{+}$with just two sigma bonds and $\mathrm{C}-\mathrm{C}-\mathrm{C}$ in which the central $\mathrm{C}$ is formally $\mathrm{C}^{2+}$ with just two sigma bonds. Combining the chains with the icosahedra leads to three likely structures: $\left(\mathrm{B}_{11} \mathrm{C}_{p}\right) \mathrm{CBC},\left(\mathrm{B}_{11} \mathrm{C}_{e}\right) \mathrm{CBC}$, and $\left(\mathrm{B}_{12}\right) \mathrm{CCC}$, where $\mathrm{C}_{p}$ represents the carbon on the polar site and $\mathrm{C}_{e}$ represents the carbon on the equatorial site. DFT (PBE flavor) calculations show that $\left(\mathrm{B}_{11} \mathrm{C}_{p}\right) \mathrm{CBC}$ is the ground state, with $\left(\mathrm{B}_{11} \mathrm{C}_{e}\right) \mathrm{CBC} 0.54 \mathrm{eV} /$ unit cell higher, and (B12)CCC $1.09 \mathrm{eV} /$ unit cell higher as shown in Fig. S2 of the SM [26]. For the ground state structure $\left(\mathrm{B}_{11} \mathrm{C}_{p}\right) \mathrm{CBC}$, all bonds in the chain and the exopolyhedral bonds of the $\mathrm{B}_{11} \mathrm{C}$ unit can be considered as two-centertwo-electron $(2 c-2 e)$ bonds. Thus, the boron $\mathrm{B}$ in the linear $\mathrm{C}-\mathrm{B}-\mathrm{C}$ chain must transfer one of its electrons, becoming $\mathrm{B}^{+}$. This is compatible with the $\left(\mathrm{B}_{11} \mathrm{C}_{p}\right)$ cage, which requires one additional electron to stabilize the icosahedral structure (26 skeleton bonding electrons, based on Wade's rule $[28,29])$. This leads to a representation as $\left(\mathrm{B}_{11} \mathrm{C}\right)^{1-}\left(\mathrm{C}-\mathrm{B}^{+}-\mathrm{C}\right)$ for the $\mathrm{B}_{4} \mathrm{C}$ ground state structure.

All periodic calculations were performed with the VASP package [30-32], using the PBE functional and the projector augmented wave method to account for the core-valence interactions [33]. We found that a kinetic energy cutoff of $500 \mathrm{eV}$ for the plane wave expansions gives excellent convergence of the total energies, energy differences, and structural parameters. Reciprocal space was sampled using the $\Gamma$-centered Monkhorst-Pack scheme with a fine resolution less than $2 \pi \times 1 / 60 \AA^{-1}$ except for the shear simulations. The convergence criteria were set to $1 \times 10^{-6} \mathrm{eV}$ energy difference for solving for the electronic wave function and $1 \times 10^{-3} \mathrm{eV} / \AA$ force for geometry optimization. The finite cluster calculations were performed with the Jaguar package [34], using the Becke three-parameter LeeYang-Parr hybrid functional [35] at the 6-311 G** level.

To examine the mechanical response of $\mathrm{B}_{4} \mathrm{C}$ under shear deformation, we imposed strain on a particular component while allowing structure relaxation for the other five strain components [36]. The residual stresses for relaxation of these other strain directions are all less than 0.5 GPa. The stress-strain curves were computed to provide insights into the mechanisms responsible for the elastic and plastic deformation as well as to identify the fracture modes. For the shear simulations we used supercells varying from 120 to 180 atoms for various slip systems while only the gamma point was sampled in the Brillouin zone.

The substitution of carbon into the $\mathrm{B}_{12}$ icosahedron induces small distortions in the $R \overline{3} m$ symmetry [3]. For the $\left(\mathrm{B}_{11} \mathrm{C}_{p}\right) \mathrm{CBC}$ distorted rhombohedral structure, PBE gives equilibrium lattice parameters $a=5.207 \AA, b=$ $5.057 \AA, c=5.207 \AA$ and $\alpha=66.012^{\circ}, \beta=65.159^{\circ}$, $\gamma=66.012^{\circ}$, leading to a density of $\rho=2.525 \mathrm{~g} / \mathrm{cm}^{3}$, comparable to the experimental values of $a=5.171 \AA$, $\alpha=65.68^{\circ}$, and $\rho=2.518 \mathrm{~g} / \mathrm{cm}^{3}$ at $300 \mathrm{~K}$ [2]. From distortions near equilibrium we derived the elastic moduli using the Voigt-Reuss-Hill approximation [37], leading to a bulk modulus of $B=238 \mathrm{GPa}$, and a shear modulus of $G=199 \mathrm{GPa}$. We also computed the Knoop hardness using the electronegativity approach $[38,39]$, leading to a value of $31.7 \mathrm{GPa}$. These results are in good agreement with previous calculations and experimental data [2,3,40-42]. The equations of states for various $\mathrm{B}_{4} \mathrm{C}$ stoichiometric structures are shown in Fig. S2 of the SM [26]. We find that the $\left(\mathrm{B}_{11} \mathrm{C}_{p}\right) \mathrm{CBC}$ structure is the ground state structure, consistent with previous DFT results [3].

To understand the shear-induced amorphous band formation in boron carbide, we examined the shear strength at $0 \mathrm{~K}$ of 11 slip systems. In particular, we examined (a) the two amorphous slip systems of $(01 \overline{1} \overline{1}) /\langle\overline{1} 101\rangle$ and $(11 \overline{2} 3) /\langle 1 \overline{2} 10\rangle$, which recent experiments [6,19] show form amorphous bands along $(01 \overline{1} \overline{1})$ and (11 23$)$ planes, respectively, (b) the three proposed slip systems of $(0001) /\langle\overline{1} 010\rangle$, $(0001) /\langle 10 \overline{1} 0\rangle$, and $(10 \overline{1} 1) /\langle 12 \overline{3} \overline{1}\rangle$ that form twin defects on the (0001) and (1011) planes [43,44], (c) one cleavage plane slip of $(1100) /\langle 1 \overline{1} 00\rangle$ from the depressurization amorphization experiments of single-crystal boron carbide [8], (d) the three typical hcp slip systems of $(0001) /\langle 11 \overline{2} 0\rangle$, $(10 \overline{1} 0) /\langle 11 \overline{2} 0\rangle$, and $(10 \overline{1} 1) /\langle 11 \overline{2} 0\rangle$, and (e) two arbitrary slip systems of $(0001) /\langle 01 \overline{1} 0\rangle$ and $(10 \overline{1} 0) /\langle 0001\rangle$, where the shear directions are perpendicular and parallel to the CBC chain, respectively.

Figure S1(b) of the SM [26] shows the shear simulation model of the $(01 \overline{1} \overline{1}) /\langle\overline{1} 101\rangle$ slip system, where the $(01 \overline{1} \overline{1})$ plane shears along the $\langle\overline{1} 101\rangle$ direction. The shear strength and critical fracture strain were derived from the stressstrain relation of the above systems as shown in Table S1 of the SM [26]. We find that the amorphous slip system $(01 \overline{1} \overline{1}) /\langle\overline{1} 101\rangle$ has the lowest shear strength among all 11 slip systems, indicating that it is the most likely to form under pressure. However, this slip plane is not a favorable dislocation slip plane [which are (0001) and (10i $\overline{1})$ planes $[6,42,43]]$; thus, it leads to formation of amorphous bands [19]. Our results agree exactly with recent 
nanoindentation experiments where shear-induced amorphization preferentially takes place along the $(01 \overline{1} \overline{1})$ plane [19]. Thus, understanding the failure modes along the $(01 \overline{1} \overline{1})$ slip system is essential to discover the origin of low toughness of boron carbide.

We find that the twin slip $(10 \overline{1} 1) /\langle 12 \overline{3} \overline{1}\rangle$ has the second lowest ideal shear strength. This may explain why the (1011) type of twin forms in $\mathrm{B}_{4} \mathrm{C}$ during hot pressed synthesis processes $[6,43,44]$.

Figure 1 displays the stress-strain relation of the five selected slip systems. Here we selected (1) the two amorphous slip system of $(01 \overline{1} \overline{1}) /\langle\overline{1} 101\rangle$ and $(11 \overline{2} 3) /\langle 1 \overline{2} 10\rangle$, (2) the twinning slip systems of $(10 \overline{1} 1) /\langle 12 \overline{3} \overline{1}\rangle$ and $(0001) /\langle 10 \overline{1} 0\rangle$, and (3) the typical hop slip system $(10 \overline{1} 1) /\langle 11 \overline{2} 0\rangle$.

The stress-strain curves for both amorphous slip systems show an abnormal ductile deformation region (above 0.1 shear strain) where the stress initially drops and then increases before failure. This indicates a two-step failure process for the amorphous slip systems. In contrast, the other slip systems lead to stress-strain curves showing an abrupt drop in stress at the point where the strain fails, indicating a one-step failure process.

To understand the nature of the two-step failure process for the $(01 \overline{1} \overline{1}) /\langle\overline{1} 101\rangle$ amorphous slip system, Fig. 2 shows the structural deformations at various strains. Figure 2 also shows the isosurface (at 0.85 ) of the electron localization function (ELF) $[45,46]$. The ELF, which ranges from 0 to 1, enables an effective and reliable analysis of covalent bonding and lone pair formation. The boroncarbon bonds between the icosahedra stretch continuously under shearing, as shown in Fig. S3 of the SM [26], but they do not break at the strain of 0.209 that corresponds to the shear strength. The ELF in Fig. 2(a) shows that the $\mathrm{B}-\mathrm{C}$ covalent bond between icosahedra still exists. As the strain increases to 0.245 [Fig. 2(b)], the ELF of the B-C

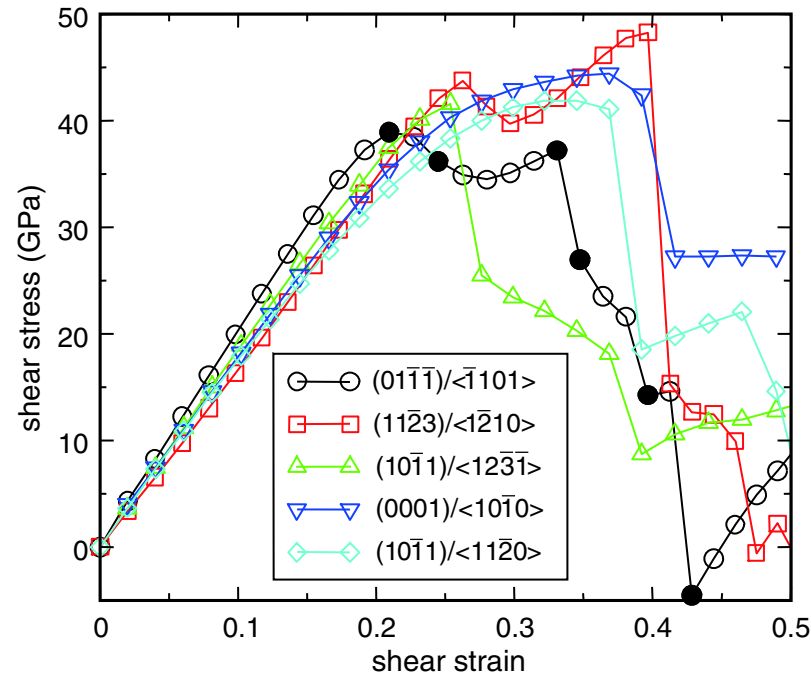

FIG. 1 (color online). The stress-strain relations for five selected slip systems under shear. The filled black circles represent the key failure steps that are examined in Figs. 2 and 3. bond moves totally to the icosahedral carbon indicating that the B-C bond is fully broken. The ELF in the icosahedral carbon shows lone pair formation. After breaking the B-C bond, the icosahedron has ten $2 c-2 e$ exopolyhedral bonds and 26 electrons for skeleton bonding (considering the one additional electron from the $\mathrm{CBC}$ chain). Thus, the icosahedral carbon atom has a lone pair pointing in the previous $\mathrm{B}-\mathrm{C}$ bond direction, corresponding to a singlet carbene. With further strain the $\mathrm{CBC}$ chain moves closer to the carbene up to a strain of 0.331 . Finally, at 0.348 strain, the middle $\mathrm{B}$ in the $\mathrm{CBC}$ chain forms a Lewis acid-Lewis base bond to the $\mathrm{C}$ atom in the icosahedron, leading to a sharp drop in the shear stress. Finally, continued shear destroys the icosahedral structures, fully relaxing the shear stress.

To get a better understanding of the failure process, we examined the deformation of one icosahedron in the crystal structure after the B is bonded to the carbene as shown in Fig. 3. We also included four of the $\mathrm{CBC}$ chains attached to the icosahedron. Here, we selected only the four CBC chains related to the destruction of icosahedron (each icosahedron is bonded to six CBC chains). At the strain of 0.331 , the icosahedron is deformed due to the shear, but is still not broken. As the strain increases to 0.348 , the middle chain boron bonds to the carbene, reducing the bond orders of the five icosahedral boron atoms bonded to this carbon, stretching them in the chain direction, which in turn increases the intracage bonds: $\mathrm{B} 1-\mathrm{B} 2$ bond from 1.84 to $2.02 \AA$, B1-B3 from 2.08 to $2.57 \AA$, as shown in Fig. 3(b). This leads to destruction of the icosahedron with a decrease in shear stress. At 0.397 strain, an additional boron in the breaking icosahedron bonds to the chain carbon, giving the chain carbon five bonds, which decreases the bond orders of the previous bonds. This weakens and breaks the previous B-C chain bond at 0.429 strain. Previously, it was proposed that shear deformation along $(01 \overline{1} \overline{1})$ would preferentially take place along this plane by the deformation and debonding of the three-atom chains [19]. However, our results find a different mechanism in which the shear deformation is mainly related to the interaction of the $\mathrm{CBC}$ chain with the carbene formed by the previous breaking of the $\mathrm{B}-\mathrm{C}$ bond between icosahedra.

To investigate the chemical nature of the failure process, we selected one icosahedron from the crystal under various strains and terminated the exopolyhedral bonds with hydrogen atoms to form a finite cluster model. This leads to the $\left(\mathrm{B}_{11} \mathrm{CH}_{12}\right)^{1-}$ model for the unstrained state, the $\left(\mathrm{B}_{11} \mathrm{CH}_{10}\right)^{1-}$ model for 0.331 strain where the carbene forms, and the $\left(\mathrm{B}_{11} \mathrm{CH}_{11}\right)^{0}$ model for 0.348 strain, where the Lewis acid-Lewis base bond forms. Here, we fixed the icosahedron atoms and relaxed the hydrogen atoms. The calculated molecular orbitals HOMO and LUMO for these models are shown in Fig. S4 of the SM [26]. For the unstrained structure, the HOMO shows the tangential character indicating a $\pi$-type interaction of the icosahedron to the CBC chain or to other icosahedra. The HOMO-LUMO gap is $8.14 \mathrm{eV}$ for the unstrained status. 


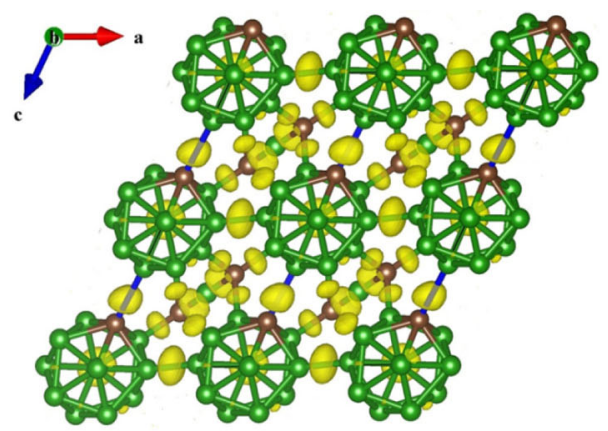

(a) Strain $=0.209$

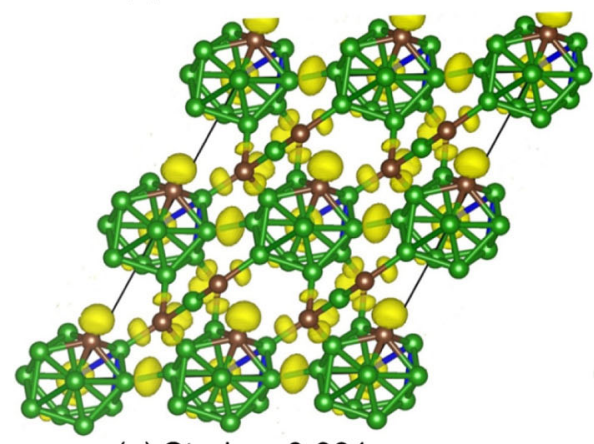

(c) Strain $=0.331$

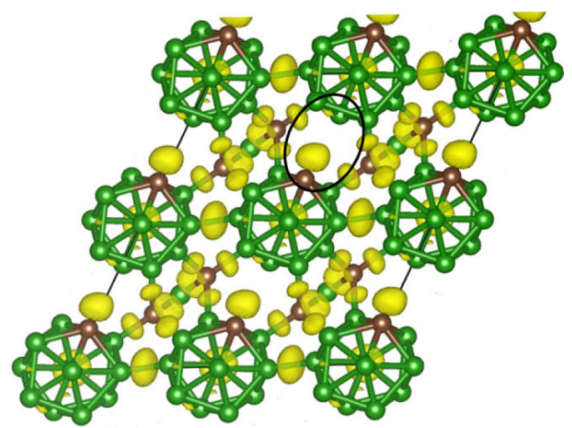

(b) Strain $=0.245$

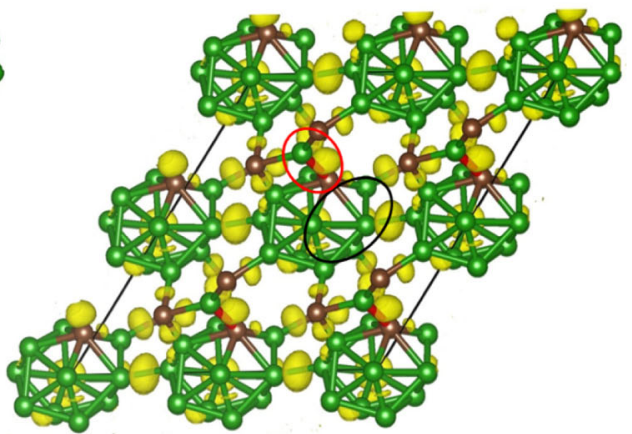

(d) Strain $=0.348$

FIG. 2 (color online). The calculated isosurfaces of the electron localization function (ELF) and structures of $\left(\mathrm{B}_{11} \mathrm{C}_{p}\right) \mathrm{CBC}$ along the $(01 \overline{1} \overline{1}) /\langle\overline{1} 101\rangle$ slip system: (a) the strain of 0.209 corresponds to the shear strength, (b) the strain of 0.245 where the B-C bond between icosahedra is totally broken leading to formation of a lone pair on the carbon, (c) the critical failure strain of 0.331 , (d) the strain of 0.348 where failure starts by the carbon lone pair forming a Lewis baseLewis acid bond to the middle chain boron. The circles highlight the key region at which changes occur. The boron and carbon atoms are represented by the green and sienna balls, respectively. The blue line indicates the future broken bonds.

At the strain of 0.331 where the carbene forms but has not reacted with the chain $\mathrm{B}$, the HOMO shows obvious radial character on the icosahedral carbon atom, indicating very reactive character. At this point the HOMO-LUMO gap is decreased to $3.03 \mathrm{eV}$. Finally at the strain of 0.348 the Lewis acid-Lewis base bond forms and the HOMO goes back to the dominantly tangential character. However, the molecular orbital in the broken icosahedron region is more prominent. Here, the HOMO-LUMO gap is increased to $3.74 \mathrm{eV}$.

Inspired by the shear band formation in metallic glass [47], we propose that the amorphous band formation in $\mathrm{B}_{4} \mathrm{C}$ proceeds as follows. First, local failure happens by breaking of an intericosahedral B-C bond, leading to a "shear transformation zone" (STZ). Here, we use STZ to describe a local failure structure that accommodates the applied shear strain. The STZ involves localized structures that undergo intense distortion from an initial to final equilibrium position through an intermediate activated state of high energy. Then, as continued propagation of this applied shear strain occurs, one STZ creates a localized distortion of the surrounding material, which triggers the formation of large planar bands of STZs, that is the "amorphous bands."

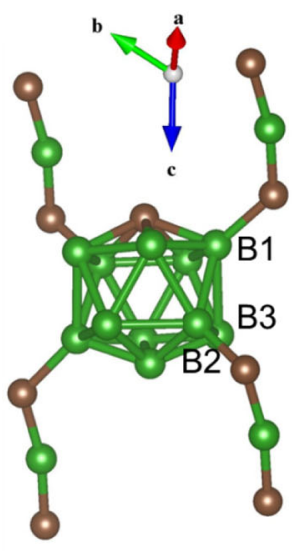

(a) Strain $=0.331$

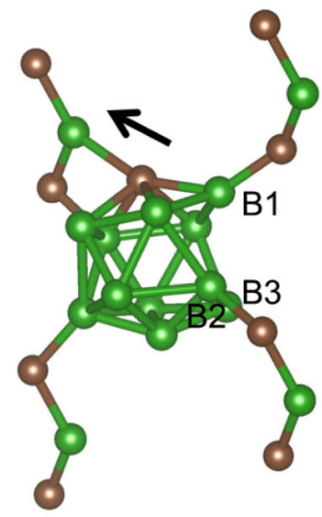

(b) Strain $=0.348$

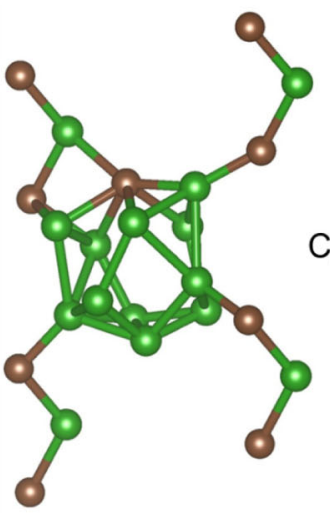

(c) Strain $=0.397$

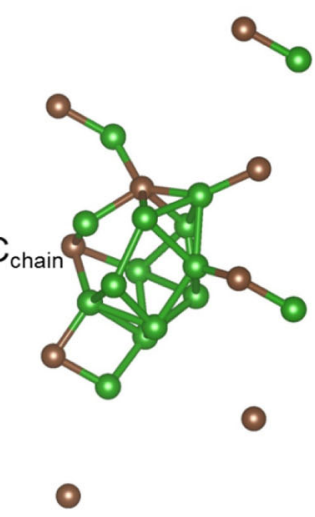

(d) Strain $=0.429$

FIG. 3 (color online). The structural evolution in the failure process shearing along the $(01 \overline{1} \overline{1}) /\langle\overline{1} 101\rangle$ slip system (here we show just one icosahedron and four CBC chains): (a) the critical failure strain of 0.331 , (b) the stain of 0.348 where the middle chain boron reacts with the carbon lone pair, stretching (represented by the arrow) and deconstructing the icosahedron, (c) the strain of 0.397 where the chain carbon is bonded to two B in the broken icosahedron, (d) the strain of 0.429 where the B-C bond in the chain is broken. The boron and carbon atoms are represented by the green and sienna balls, respectively. 
In summary, we investigated the shear-induced amorphous band formation in $\mathrm{B}_{4} \mathrm{C}$. The shear strength for the slip system $(01 \overline{1} \overline{1}) /\langle\overline{1} 101\rangle$ is the lowest among all 11 slip systems. The activation of this slip system under stress leads to amorphous band formation. The atomistic description of the failure process is as follows: first, the intericosahedron B-C bond breaks, leading to carbene formation (Lewis base), then the carbene reacts with the middle boron in the chain (Lewis acid), which in turn withdraws electrons from the icosahedron leading to its destruction. This demonstrates a unique failure process of $\mathrm{B}_{4} \mathrm{C}$, leading to amorphous band formation. Since the other two stable structures for $\mathrm{B}_{4} \mathrm{C}$ : $\left(\mathrm{B}_{11} \mathrm{C}_{e}\right) \mathrm{CBC}(0.54 \mathrm{eV}$ less stable $)$ and $\left(\mathrm{B}_{12}\right) \mathrm{CCC}(1.09 \mathrm{eV}$ less stable) have no intericosahedral B-C bond, we expect them not to exhibit this failure mode. This suggests that modifying the synthetic processes to minimize $\left(\mathrm{B}_{11} \mathrm{C}_{p}\right) \mathrm{CBC}$ character in favor of the other two could improve the strength of the materials. Since each phase has distinguishable Raman and IR spectra, one might be able to rapidly optimize these synthetic conditions.

This work was supported by the Defense Advanced Research Projects Agency (W31P4Q-13-1-0010, program manager, Judah Goldwasser). In addition some support was provided by the Army Research Laboratory under Cooperative Agreement No. W911NF-12-2-0022. We thank Dr. Wei-Guang Liu at Caltech for the useful discussions.

* Corresponding author. wag@wag.caltech.edu

[1] A. O. Sezer and J. I. Brand, Mater. Sci. Eng. B 79, 191 (2001).

[2] F. Thevenot, J. Eur. Ceram. Soc. 6, 205 (1990).

[3] V. Domnich, S. Reynaud, R. A. Haber, and M. Chhowalla, J. Am. Ceram. Soc. 94, 3605 (2011).

[4] J. L. Hoard and R. E. Hughes, in The Chemistry of Boron and Its Compounds, edited by E. L. Muetterties (Wiley, New York, 1967).

[5] A. K. Suri, C. Subramanian, J. K. Sonber, and T. S. R. Ch. Murthy, Int. Mater. Rev. 55, 4 (2010).

[6] M. W. Chen et al., Science 299, 1563 (2003).

[7] G. Fanchini, J. W. McCauley, and M. Chhowalla, Phys. Rev. Lett. 97, 035502 (2006).

[8] X. Q. Yan, Z. Tang, L. Zhang, J. J. Guo, C. Q. Jin, Y. Zhang, T. Goto, J. W. McCauley, and M. W. Chen, Phys. Rev. Lett. 102, 075505 (2009).

[9] K. Shirai, J. Superhard Mater. 32, 205 (2010).

[10] K. Shirai, J. Superhard Mater. 32, 336 (2010).

[11] D. Emin, J. Solid State Chem. 179, 2791 (2006).

[12] K. N. Bourne, Proc. R. Soc. A 458, 1999 (2002).

[13] G. R. Johnson and T. J. Holmquist, J. Appl. Phys. 85, 8060 (1999).

[14] T. Mashimo and M. Uchino, J. Appl. Phys. 81, 7064 (1997).

[15] T. J. Vogler et al., J. Appl. Phys. 95, 4173 (2004).

[16] V. Domnich, Y. Gogotsi, M. Trenary, and T. Tanaka, Appl. Phys. Lett. 81, 3783 (2002).

[17] X. Q. Yan, W. J. Li, T. Goto, and M. W. Chen, Appl. Phys. Lett. 88, 131905 (2006).
[18] M. W. Chen and J.W. McCauley, J. Appl. Phys. 100, 123517 (2006).

[19] K. M. Reddy, P. Liu, A. Hirata, T. Fujita, and M. W. Chen, Nat. Commun. 4, 2483 (2013).

[20] K. M. Reddy, A. Hirata, P. Liu, T. Fujita, T. Goto, and M. W. Chen, Scr. Mater. 76, 9 (2014).

[21] Q. An, W. A. Goddard III, H. Xiao, and T. Cheng, Chem. Mater. 26, 4289 (2014).

[22] C. Jiang, Z. J. Lin, J. Z. Zhang, and Y. S. Zhao, Appl. Phys. Lett. 94, 191906 (2009).

[23] W. Zhou, H. Sun, and C. F. Chen, Phys. Rev. Lett. 105, 215503 (2010).

[24] Y. Zhang, H. Sun, and C. F. Chen, Phys. Rev. B 73, 144115 (2006).

[25] S. Aryal, P. Rulis, and W. Y. Ching, Phys. Rev. B 84, 184112 (2011).

[26] See Supplemental Material athttp://link.aps.org/supplemental/ 10.1103/PhysRevLett.113.095501 for the table listing the predicted shear strength and the critical failure strain for various slip systems, and the figures of the $\mathrm{B}_{4} \mathrm{C}$ structure and ideal shear simulation model; the predicted equations of state for various $\mathrm{B}_{4} \mathrm{C}$ stoichiometry structures; the bond distance of B-C between icosahedra shearing along the $(01 \overline{1} \overline{1}) /\langle\overline{1} 101\rangle$ slip system; and molecular orbital analysis at various strains for the $(01 \overline{1} \overline{1}) /\langle\overline{1} 101\rangle$ slip system.

[27] V. I. Matkovich, J. Am. Chem. Soc. 83, 1804 (1961).

[28] K. Wade, J. Chem. Soc. D 15, 792 (1971).

[29] D. M. P. Mingos, Nature (London) Phys. Sci. 236, 99 (1972).

[30] G. Kresse and J. Hafner, Phys. Rev. B 47, 558 (1993).

[31] G. Kresse and J. Furthmüller, Comput. Mater. Sci. 6, 15 (1996).

[32] G. Kresse et al., Phys. Rev. B 54, 11169 (1996).

[33] G. Kresse and D. Joubert, Phys. Rev. B 59, 1758 (1999).

[34] A. D. Bochevarov, E. Harder, T. F. Hughes, J. R. Greenwood, D. A. Braden, D. M. Philipp, D. Rinaldo, M. D. Halls, J. Zhang, R. A. Friesner, Int. J. Quantum Chem. 113, 2110 (2013)..

[35] C. T. Lee, W. T. Yang, and R. G. Parr, Phys. Rev. B 37, 785 (1988).

[36] D. Roundy, C. R. Krenn, M. L. Cohen, and J. W. Morris, Phys. Rev. Lett. 82, 2713 (1999).

[37] H. D. Chung, Philos. Mag. 8, 833 (1963).

[38] K. Y. Li, X. T. Wang, F. F. Zhang, and D. F. Xue, Phys. Rev. Lett. 100, 235504 (2008).

[39] A. O. Lyakhov and A. R. Oganov, Phys. Rev. B 84, 092103 (2011).

[40] R. J. Nelmes, J. S. Loveday, R. M. Wilson, W. G. Marshall, J. M. Besson, S. Klotz, G. Hamel, T. L. Aselage, and S. Hull, Phys. Rev. Lett. 74, 2268 (1995).

[41] K. A. Schwetz and W. Grellner, J. Less-Common Met. 82, 37 (1981).

[42] S. R. Murthy, J. Mater. Sci. Lett. 4, 603 (1985).

[43] Y. Li, Y.H. Zhao, W. Liu, Z.H. Zhang, R. G. Vogt, E. J. Lavernia, and J. M. Schoenung, Philos. Mag. 90, 783 (2010).

[44] F.-F. Xu and Y. Bando, J. Phys. Chem. B 108, 7651 (2004).

[45] A. D. Becke and K. E. Edgecombe, J. Chem. Phys. 92, 5397 (1990).

[46] B. Silvi and A. Savin, Nature (London) 371, 683 (1994).

[47] A. Argon, Acta Metall. 27, 47 (1979). 